\title{
JASPLAKINOLIDE, AN ACTIN STABILIZING AGENT, ALTERS ANAPHASE CHROMOSOME MOVEMENTS IN CRANE-FLY SPERMATOCYTES.
}

\author{
by: $\quad$ Lele Xie and Arthur Forer ${ }^{1}$
}

1: Author for correspondence:

Arthur Forer

Biology Department

York University

Toronto, Ontario

Canada M3J 1P3

Email: aforer@yorku.ca

Telephone: (416) 736-5398

Key Words: Chromosome movement, crane fly spermatocytes, actin, 


\begin{abstract}
We added jasplakinolide to anaphase crane-fly spermatocytes and determined its effects on chromosome movement. Previous work showed that actin depolymerizing agents such as cytochalasin D or latrunculin B blocked or slowed chromosome movements; we wanted to compare the effects of jasplakinolide, a compound that stabilizes actin filaments against depolymerization. Jasplakinolide had the same effect on movements of each half-bivalent in a separating pair of halfbivalents, but different half-bivalent pairs in the same cell often responded differently, even when the concentrations of jasplakinolide varied by a factor of two. Jasplakinolide had no effect on about $20 \%$ of the pairs, but otherwise caused movements to slow, or to stop, or, rarely, to accelerate. When cells were kept in jasplakinolide, stopped pairs eventually resumed movement; slowed pairs did not change their speeds. Confocal microscopy indicated that neither the distributions of spindle actin filaments nor the distributions of spindle microtubules were altered by the jasplakinolide. It is possible that jasplakinolide binds to spindle actin and blocks critical binding sites, but we suggest that jasplakinolide affects anaphase chromosome movement by preventing actin-filament depolymerization that is necessary for anaphase to proceed. Overall, our data indicate that actin is involved in one of the redundant mechanisms cells use to move chromosomes.
\end{abstract}




\section{INTRODUCTION}

Microtubules and their associated enzymes and motor proteins generally are considered to be the major or the only players in the formation of mitotic spindles and the major players in producing the forces on the chromosomes that move them to the equator during prometaphase, that keep them at the equator during metaphase, and that move them to the poles during anaphase (e.g., Rogers et al., 2004; Civelekoglu-Scholey et al., 2006; McNally et al., 2006; O’Connell and Khodjakov, 2007; Yang et al., 2007; Bannigan et al., 2008). However, various data indicate that a variety of other components are important for spindle formation and for chromosome movement; these include spindle matrix components (review: Johansen and Johansen, 2007) and actin, myosin and other “muscle” proteins (Fabian et al., 2007a; Forer et al., 2008).

Various physiological, morphological and molecular data suggest that a spindle matrix functions in setting up the mitotic spindle and in propelling chromosomes poleward (e.g., Johansen and Johansen, 2007; Forer et al., 2008). For example, severing a kinetochore fibre during metaphase does not reduce the poleward force acting on the metaphase chromosome, implying that an underlying spindle matrix produces poleward force on the remnant kinetochore fibre stub (Maiato et al., 2004). Also, anaphase chromosomes can continue to move when their kinetochore microtubules are severed, similarly implying that an underlying spindle matrix produces poleward force on the remnant kinetochore fibre stubs (Forer et al., 2003). Further, when all the microtubules across a half-spindle are severed, the spindle poles move towards each other (Spurck et al., 1990), indicating that in the absence of microtubule connections there still 
are forces between the poles, also presumed to be produced by a spindle matrix. Most recently, genetic alterations in a spindle matrix component (Chromator) result in altered spindle shapes and in defects in chromosome segregation (Qi et al., 2007). We do not know how the spindle matrix functions, however: some consider it to be a static substrate from which motor proteins propel spindle microtubules (e.g., Scholey et al., 2001; Miyamoto et al., 2004; Mitchison et al., 2005) but the matrix also might in itself be elastic or contractile, as suggested by Pickett-Heaps et al. (1984), and the matrix may function together with non-microtubule spindle fibre components as well as with microtubules (e.g., Forer et al., 2008).

Actin/myosin involvement in mitosis has been deduced from studies that actin, myosin and titin are present in a variety of spindles (e.g., Table I of Forer et al., 2003; Robinson and Snyder, 2005; Yasuda et al., 2005; Fabian and Forer, 2007; Fabian et al., 2007b) and, also in a variety of cells, from studies of the effects of actin inhibitors, of myosin inhibitors or of genetic alterations to myosin (review in Table II of Forer et al. 2005; also Fabian and Forer, 2005; Fabian and Forer, 2007). In crane-fly spermatocytes in particular, the cells used for the experiments we describe herein, anaphase chromosome movement is altered by actin inhibitors cytochalasin D and latrunculin B, both of which cause depolymerization of actin filaments, and is altered by myosin inhibitors BDM and Y27632, both of which block myosin activity (Forer and Pickett-Heaps, 1998; Silverman-Gavrila and Forer, 2001; Fabian and Forer, 2005; Fabian et al., 2007a). The actin inhibitors cytochalasin D and latrunculin B (and others) and the myosin inhibitor BDM (Forer and Fabian, 2005) also block tubulin flux in kinetochore fibres (Silverman-Gavrila and Forer, 2000; Forer et al., 2007), further implicating them as important players during mitosis. 
Myosin has been implicated further by treating anaphase cells with a drug, calyculin A, that enhances myosin activity. Experiments cited above show that chromosome movements are stopped or slowed when myosin is inhibited, but what if myosin activity is enhanced? Myosin is active only when a regulatory light chain is phosphorylated; the level of phosphorylation is in homeostasis because of a balance between the phosphorylation of myosin by myosin light chain kinase or other phosphorylating enzymes and the dephosphorylation of myosin by myosin phosphatase. By blocking myosin phosphatase with calyculin, the myosin becomes hyperphosphorylated and hyperactive: enhancing myosin in this way causes anaphase movements to speed up (Fabian et al., 2007a). Thus, compounds that block myosin activity or depolymerize actin filaments block or slow chromosome movement, whereas calyculin A, that enhances myosin activity, speeds up chromosome movement. These complementary kinds of results indicate that myosin is involved in propulsion of anaphase chromosomes.

In the present experiments we describe the effects of jasplakinolide on chromosome movements in crane-fly spermatocytes. The anti-actin agents used previously to slow or stop chromosome movements, cytochalasin D and latrunculin B, cause depolymerization of spindle actin filaments (Silverman-Gavrila and Forer, 2000; Fabian and Forer, 2005). Jasplakinolide, on the other hand, stabilizes existing actin filaments and, at higher concentrations (Cramer, 1999), also can cause polymerization of additional actin filaments or cause bundling of pre-existing actin filaments (Holzinger, 2001; Terada et al., 2000), without affecting the structure or function of nearby microtubules (Holzinger, 2001). Jasplakinolide should stabilize spindle actin filaments and prevent their depolymerization. If actin filaments are involved with force production, 
jasplakinolide would alter chromosome movement if the spindle actin filaments need to be depolymerized during anaphase. Jasplakinolide also could alter chromosome movement if the jasplakinolide that is bound to actin interfered with the binding sites of proteins that interact with actin. As we report herein, jasplakinolide indeed alters anaphase chromosome movements in crane-fly spermatocytes.

\section{MATERIALS AND METHODS}

Living cell preparations: We removed testes from IV instar larvae of crane flies (Nephrotoma suturalis Loew) under Halocarbon oil (Halocarbon Products Corp., River Edge, N.J.), rinsed them with insect Ringer solution $(0.13 \mathrm{M} \mathrm{NaCl}, 0.005 \mathrm{M} \mathrm{KCl}, 0.001 \mathrm{M} \mathrm{CaCl}, 0.02$ M Na2HPO4- $\mathrm{KH}_{2} \mathrm{PO} 4$ buffer, $\mathrm{pH}$ 6.9), and put the cells into a fibrin clot as described in Forer and Pickett-Heaps (2005). Briefly, cells were spread on a coverslip in fibrinogen (Sigma) that was dissolved in insect Ringer solution; then we added thrombin to form a fibrin clot. Once the preparation was mounted in a perfusion chamber, the cells were perfused with insect Ringer solution or with insect Ringer solution that contained jasplakinolide (Alexis Biochemicals or Invitrogen). We dissolved the jasplakinolide in dimethylsulphoxide (DMSO) at a concentration of $0.56 \mathrm{mM}$ and kept measured aliquots at $-80^{\circ} \mathrm{C}$. On the day of the experiment we diluted the stock japlakinolide in insect Ringer solution, to reach final concentrations of $0.56 \mu \mathrm{M}$ or $1.12 \mu \mathrm{M}$, and perfused the cells with this jasplakinolide. As reported previously (Fabian and Forer, 2007; Forer and Pickett-Heaps, 1998; 2005; La Fountain et al., 1992; 2001), perfusion itself with insect 
Ringer solution or with insect Ringer solution that contained 0.2\% DMSO (the maximum amount in our japlakinolide solution) had no effect on anaphase or on progression of the cells into anaphase or on anything that we have measured.

Microscopy and data analysis. We observed cells using a Nikon oil immersion phasecontrast lens $(\mathrm{NA}=1.30)$ and recorded phase-contrast images on DVDs in real time. We digitized and time-lapsed the images using Virtual Dub [available from http:// fcchandler.home.comcast.net] and analyzed them as in Fabian and Forer (2007).

Fluorescence Staining. We used procedures basically as decribed previously (Fabian and Forer, 2005; Fabian et al., 2007b). Briefly, we lysed treated or control cells in a lysis buffer containing 5\% DMSO and 1\% Nonidet P-40. After 15 minutes the cells were fixed for $5 \mathrm{~min}$ in 0.25\% glutaraldehyde in PBS (phosphate buffered saline: $0.13 \mathrm{M} \mathrm{NaCl}, 6 \mathrm{mM}$ phosphate buffer, $\mathrm{pH}$ 7); rinsed 5 min with PBS; incubated for $10 \mathrm{~min}$. in $1 \mathrm{mg} / \mathrm{ml}$ sodium borohydride $\left(\mathrm{NaBH}_{4}\right)$ in PBS; rinsed for 5 min in PBS; and stored in 1:1 PBS/glycerol at 4/C until staining.

To stain the cells, we washed off the glycerol, rinsed the coverslips in PBS, rinsed them in PBS containing $0.2 \%$ Triton- $\mathrm{X}$, and then added $1.45 \mu \mathrm{M}$ Alexa 488 phalloidin (Molecular Probes/Invitrogen). After the phalloidin incubation and after each subsequent addition of antibody, before proceeding with the next step the coverslips were rinsed twice in PBS and then once in PBS containing Triton-X; all steps were at room temperature. After incubating the coverslips with phalloidin for 45 min in the dark, we added rat monoclonal antibody YL 1/2 (Serotec) diluted 1:100, for 45 min. Then we added Alexa 594 goat anti-rat-IgG (Molecular 
Probes/Invitrogen) diluted 1:200, and incubated for $45 \mathrm{~min}$. After final rinses we mounted the coverslips on slides in a solution of Mowiol (Calbiochem), prepared essentially as described by Osborn and Weber (1982), to which we added 500 mgm/litre paraphenylene diamine (Sigma) as antifading agent. We left the slides overnight in a fume hood, in the dark, for the Mowiol to harden, and then we stored them at $4^{\circ} \mathrm{C}$ in the dark, until we studied them using confocal microscopy. We obtained fluorescence images using an Olympus Fluoview confocal microscope with Olympus Plan Apo 60X oil immersion objective (numerical aperture 1.4). The images were collected with Fluoview software and further processed using Image J (public domain software available at http://rsb.info.nih.gov/ij/) and Adobe Photoshop. Figures were prepared adjusting only contrast and brightness.

\section{RESULTS}

Chromosome movements in control spermatocytes. In control cells, the three autosomal bivalents and two univalent sex chromosomes move back and forth within the spindle during prometaphase, and eventually converge to the cell equator. By metaphase both the bivalents and univalents are aligned at the equator and kinetochore fibres connect each bivalent and each univalent to both poles. During anaphase, partner half-bivalents move poleward, for 10-20 minutes, with separation velocities that at $20-25 / \mathrm{C}$ range from $0.5 \mu \mathrm{m} / \mathrm{min}$ to $3 \mu \mathrm{m} / \mathrm{min}$ (Schaap and Forer, 1979), as illustrated in Fig. 1 and Fig 2. The sex-chromosome univalents stay at the 
metaphase plate until the half-bivalents near the poles; they move to opposite poles starting about 15-30 mins after onset of autosomal anaphase, and as each univalent moves poleward the leading kinetochore fibre shortens and the trailing kinetochore fibre elongates. Cytokinesis starts about 20-35 minutes after onset of autosomal anaphase.

Effects of jasplakinolide applied during anaphase. Jasplakinolide altered chromosome movement when applied during autosomal anaphase. We treated cells shortly after anaphase onset and studied the effects on autosome movement both by visual observation of the time-lapsed movies and by plotting graphs of chromosome separation versus time. We could not distinguish between the effects of $0.56 \mu \mathrm{M}$ jasplakinolide and of $1.12 \mu \mathrm{M}$ jasplakinolide, so we pooled the results.

Both chromosomal half-bivalents in each separating pair were affected the same by the jasplakinolide, but different pairs in the same cell were not necessarily affected in the same way. In some cells not all the half-bivalent pairs were affected by the drug. Of the 24 cells studied, different pairs in the same cell responded differently in 15 cells, and there were only two cells in which no separating pairs were affected. We did not always follow all 3 separating autosomal pairs in individual cells because often one pair was in a different focal plane than the others. Hence we studied only 53 separating pairs in the 24 cells, fewer than the 72 possible. Jasplakinolide altered the movements of 42 of the 53 pairs, i.e., of more than three-quarters of the pairs: within 3-4 minutes after adding jasplakinolide the chromosomes either slowed, stopped, or, rarely, sped up, as listed in Table I and as illustrated in Figures 3 and 4. Slowed chromosome 
pairs were slowed to varying extents, from 30-80\% (Figure 5), the percentage of slowing perhaps being a function of initial separation velocity (Figure 6).

Some cells remained in jasplakinolide throughout anaphase. When chromosomes had been stopped by the jasplakinolide, their movements eventually resumed in the continued presence of jasplakinolide (e.g., Fig. 4B). When chromosomes had been slowed by the jasplakinolide, on the other hand, chromosome movements remained at the slowed velocity, without any recovery (Table II).

Jasplakinolide was washed out from other cells by perfusion with insect Ringer solution, at times ranging from 5 to 10 minutes after the initial treatment. When chromosomes had been stopped by the jasplakinolide, their poleward movements resumed within 3-4 minutes after the jasplakinolide was washed out (e.g., Fig. 4C), at separation velocities that often were slower than the initial velocities (Figure 7). When chromosomes had been slowed by the jasplakinolide, on the other hand, the washout had little effect, and poleward motions generally continued with the same slowed velocities (Table III, e.g., Fig. 4A).

Effects of jasplakinolide applied before anaphase. We added jasplakinolide to cells in prometaphase and metaphase, at times ranging from 3.5 to 33 minutes before anaphase onset, and we followed subsequent anaphase movements. As with treatment during anaphase, different half-bivalent pairs in a cell did not necessarily respond the same. We followed 21 half-bivalent pairs in 10 cells: movements were normal; or movements stopped (4 pairs) after

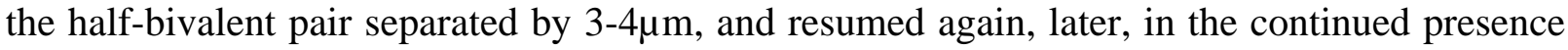
of jasplakinolide; or movements slowed (3 pairs) after the half-bivalent pair separated 3-4 $\mu$ m. 
One major difference between these results and results from jasplakinolide added after anaphase onset is the frequency of effects: when jasplakinolide was added prior to anaphase, autosome movements were altered only one-third of the time, whereas when jasplakinolide was added during anaphase, autosome movements were altered more than three-quarters of the time (Table IV). Another difference is that when jasplakinolide was added prior to anaphase, in most cells (8/10) different pairs in the cell responded the same, whereas when jasplakinolide was added during anaphase, in most cells (15/24) different pairs in the cell responded differently. The effects of jasplakinolide did not seem to vary with time in prometaphase when the drug was added (Figure 8), but we probably need more data to be certain of this point.

Effects of jaslakinolide on spindle actin. In previous work, crane-fly spermatocytes were treated with 300nM jasplakinolide for 10 minutes and metaphase cells were studied to see if there were changes in the distribution of actin filaments or if there were changes in the acetylation pattern or distribution of spindle microtubules: there were no changes in spindle actin or microtubules, but actin filaments outside the spindle area were possibly more prominent or thicker than in control cells (Silverman-Gavrila and Forer, 2000). This suggests that the jasplakinolide alters actin filament dynamics without gross changes in actin filament distribution. To substantiate this conclusion we felt that we should look at anaphase cells treated with $1.2 \mu \mathrm{M}$ jasplakinolide, the maximum concentration we used to study anaphase chromosome movements, and to look at cells treated for 5 minutes, since we saw altered movement either immediately upon adding jasplakinolide or up to 3 minutes after adding jasplakinolide. Actin in control cells, not treated with jasplakinolide, looked as described 
previously (Figure 9; also Czaban and Forer, 1994; Silverman-Gavrila and Forer, 2000; Forer et al., 2003; Fabian and Forer, 2005; Fabian et al., 2007a), as did actin in cells treated with jasplakinolide (Fig. 10). The only possible difference we saw in the jasplakinolide treated metaphase and anaphase cells is that the kinetochore bundles of actin filaments perhaps seemed more prominent, or sturdy-looking than in control cells (Figs. 9, 10). Thus, jasplakinolide does not change the distribution or appearance of spindle microtubules or spindle actin.

\section{DISCUSSION}

We treated crane-fly spermatocytes with $0.56 \mu \mathrm{M}$ or $1.12 \mu \mathrm{M}$ jasplakinolide, a compound that stabilizes existing actin filaments, that sometimes bundles existing actin filaments, and that can promote polymerization of new filaments (e.g., Holzinger and Meindl, 1997; Terada et al., 2000; Holzinger, 2001). In our experiments, after jasplakinolide treatment the spindle F-actin appeared the same as in controls, or, if anything, more robust than in controls (Figure 10), consistent with experiments that show that $1 \mu \mathrm{M}$ jasplakinolide does not perturb actin cytoskeleton organization in PtK cells for the first 15-20 minutes of treatment (Cramer, 1999). When added during anaphase, jasplakinolide immediately slowed or stopped the poleward movements of more than $75 \%$ of the chromosome pairs (Table I). Chromosome pairs in a given cell responded differently from each other in most cells (15/24), and all pairs that stopped moving resumed movement again in the continued presence of jasplakinolide (Table II). We do not know why the chromosomes respond in pairs, but that is characteristic for these cells: separating half-bivalents respond the same to ultraviolet microbeam irradiation of 
individual kinetochore fibres and to whole cell treatment with various drugs (Forer, 1966; Forer and Pickett-Heaps, 1998; Fabian and Forer, 2005; Fabian et al., 2007a). The interdependence of half-bivalents moving to opposite poles may be a result of the 'signalling' between separating chromosomes in anaphase (Yin and Forer, 1996; Ilagan and Forer, 1997a; Wong and Forer, 2003) or may be the result of the tethers between the arms of separating half-bivalents described by LaFountain et al. (2002), that exert backwards force on chromosome arms (also see Ilagan and Forer, 1997b; Wong and Forer, 2004) and that seem to contain titin (Fabian et al., 2007b). Whatever the reason, in crane-fly spermatocytes the movements of separating halfbivalents are coordinated and partner half-bivalents respond the same, even when different pairs in the cell respond differently.

These effects of jasplakinolide treatment on chromosome movements are the same as those reported for cytochalasin D and for latrunculin B, agents that function oppositely to jasplakinolide in that they depolymerize actin filaments: cytochalasin D and latrunculin B stop or slow anaphase chromosome movement, and different chromosome pairs in the same cell often respond differently (Forer and Pickett-Heaps, 1998; Fabian and Forer, 2005), just as with jasplakinolide. How to explain the puzzling result that different chromosome pairs in the same cell respond differently to the same treatment, and how to explain that stabilizing agents give the same results as depolymerizing agents?

The difference in response for different chromosome pairs does not seem to be a matter of concentration of the drug. Variation between pairs in a cell occurs for jasplakinolide concentrations that differed by a factor of two $(0.56 \mu \mathrm{M}$ and $1.12 \mu \mathrm{M})$, and for cytochalasin D 
concentrations that differed by a factor of two, $20 \mu \mathrm{M}$ and $40 \mu \mathrm{M}$ (Forer and Pickett-Heaps, 1998). BDM, an anti-myosin agent, also has variable effects on pairs within a cell, causing slowing, stopping or having no effect, both at 20mM BDM (Silverman-Gavrila and Forer, 2001; Fabian and Forer, 2005) and at 5mM BDM (Fabian and Forer, 2005; Fabian et al., 2007a). Thus the variation in responses within a cell does not seem to be due to the drug being at a borderline concentration. What else could it be due to?

Several reports describe differences between individual kinetochore fibres in the same spindle. Some experiments have shown that microtubules in individual kinetochore fibres in a spindle can have different origins, some microtubules in the bundle being nucleated by the centrosome and others being nucleated by the chromosomes themselves, the exact status of each of the microtubules in each kinetochore fibre being due to circumstances in the cell at the time the spindle is forming (Khodjakov et al., 2003; Maiato et al., 2004; discussions in Gadde and Heald, 2004; Wadsworth and Khodjakov, 2004; O’Connell and Khodjakov, 2007; Johansen and Johansen, 2007). In other experiments, Cameron et al. (2006) found variable flux rates in different $\mathrm{PtK}_{1}$ kinetochore fibres within a single spindle. They explained the heterogeneity as "possibly associated with spatially and temporally varying contributions of multiple force-generation mechanisms” within individual spindles. Thus it does not seem much of a stretch to suggest that the different responses we observed are due to differences in the individual kinetochore fibres. Thus we assume, with Cameron et al. (2006), that there are multiple mechanisms to produce force; the possible force producers could include microtubules and a variety of microtubule motors as well as myosin (discussion in Fabian and Forer, 2005; 
Fabian et al., 2007a). For purposes of discussion here, we will consider broadly those mechanisms that use actin and those that do not use actin. Just as kinetochore fibres in a single spindle may have microtubules with different origins, depending on circumstances (e.g., Khodjakov et al., 2003; Maiato et al., 2004), we assume that different kinetochore fibres in a single spindle may have different combinations of redundant force producers, different combinations of force producers that do or do not use actin, depending on the interactions between the spindle matrix and the various force producers at the time the spindle fibre is forming. Thus, some pairs of chromosomes stop moving when anti-actin drugs (jasplakinolide, cytochalasin, latrunculin) are added: these kinetochore fibres must rely heavily on force producers that utilize actin. Other pairs are not affected by those drugs: these kinetochore fibres must not rely very much on participation of actin. Yet other pairs are slowed: these fibres must have several mechanisms that participate at the same time, actin and non-actin mechanisms, that participate together to varying degrees. Chromosome pairs that stopped moving start up again in the continued presence of the anti-actin drug: these fibres must have the ability to switch to an alternate, non-actin mechanism after the actin is depolymerized.

By extending this line of thinking we can offer an explanation for why jasplakinolide treatment before anaphase is different from treatment after anaphase: when added before anaphase there were fewer effects (Table IV) and the chromosome pairs in the same cell tended to respond the same ( $8 / 10$ cells). We think this is because of the redundancies in force producers. When one removes actin prior to the spindle being formed, the cell adapts to the absence of actin by utilising redundant, solely non-actin force producers. Thus, depending 
when the drug is added during formation of the fibre, some fibres may be more completely formed than others, and those not completely formed can adapt to the absence of one component by utilising a different, redundant force system. In general the earlier the drug is added prior to anaphase there will be less and less of an effect on movement and more homogeneity in the different fibres and in responses to drugs since one source of variability is removed, exactly what we have found. In related experiments that follow a similar line of reasoning, even though latrunculin B applied during anaphase blocks/slows chromosome movement, anaphase is normal when latrunculin B is applied many minutes before functional anaphase kinetochore fibres are established, in early prometaphase, and the cell is kept in latrunculin B (Fabian and Forer, 2005): actin-filament-free spindles function normally when the spindle forms in the absence of functional actin, but actin filaments ordinarily are present and when they are present the cell utilizes them as part of the spindle. In related findings, when an ultraviolet microbeam irradiates a portion of an individual kinetochore fibre in anaphase crane-fly spermatocytes, the irradiated half-bivalent pair acts independently from the nonirradiated pairs in that the irradiated pair stops moving but the others move normally (Forer, 1966). When a single kinetochore fibre is irradiated in metaphase, however, shortly before anaphase onset, then all chromosomes respond the same (Forer, 1966): at anaphase all disjoin into half-bivalents but all stop moving, and do not move poleward. If our interpretation above is correct, these data would seem to indicate that the spindle acts as a single functional unit until the spindle is fully set up to move chromosomes to the poles in anaphase. Thus, prior to 
completion of a functional spindle, disruption of a single element in the tensegral matrix of forces effects all chromosomes.

Why does the actin-stabilizing agent jasplakinolide have the same effects as the actin depolymerizing agents? One possibility is that the jasplakinolide affects movement by blocking crucial binding sites on the actin filaments. Another possibility is the interpretation favoured in other experiments, that actin filaments need to be slowly depolymerized.. For example, lamellipodial protrusions in PtK cells are inhibited by jasplakinolide; this was taken to mean that actin-filament disassembly is required for protrusion of lamellipodia and jasplakinolide inhibitd protrusioin by stabilizing the filaments against depolymerization. In other work, after nuclear membrane breakdown chromosomes in starfish oocytes move to the position of previously formed asters; jasplakinolide prevents that movement (Lénárt et al., 2005), as does cytochalasin D. Lénárt et al. (2005) argued that because actin filaments are necessary for chromosomes to move, chromosomes do not move when the actin filaments are depolymerized by cytochalasin $\mathrm{D}$. They argue further that the actin filament network changes shape (“contraction”) by slow, controlled depolymerization, hence chromosome movement is blocked when filaments are stabilized by the jasplakinolide. The same could apply to chromosome movement in anaphase crane-fly spermatocytes. That is, kinetochore fibre force producers can utilize actin, so depolymerization of actin with cytochalasin or latrunculin alters anaphase chromosome movement. The filamentous actin in kinetochore fibres undergoes slow, controlled depolymerization during anaphase; jasplakinolide stabilizes the filaments and blocks their depolymerization and hence alters anaphase chromosome movement. Our present data do 
not distinguish between the two possible explanations for why jasplakinolide alters chromosome movement. However, kinetochore fibres contain actin filaments throughout anaphase (e.g., Fig. 9; also Silverman-gavrila and Forer, 2003; Fabian and Forer, 2007b), as the kinetochore fibre shortens, the lengths of the actin filaments also shorten so the effects of jasplakinolide on anaphase movements might be because the spindle actin filaments are stabilized and unable to depolymerize during anaphase.

Conclusions. Jasplakinolide, a compound that stabilizes actin filaments, slows or stops anaphase chromosome movement. Agents that depolymerize actin filaments have the same effects on anaphase chromosome movements (e.g., Forer and Pickett-Heaps, 1998). The two kinds of experiments together indicate that chromosome movements normally require actin filaments. There are vaious ways that actin might be involved, one of which is illustrated in Forer et al. (2008), but at present we don't really know what actin (and its motors) do during anaphase, except that they are utilized somehow as one of the redundant force production options.

ACKNOWLEDGEMENTS. This work was supported by grants from the Natural Sciences and Engineering Research Council of Canada to A.F.. We thank Jeremy PickettHeaps and Lacramiora Fabian for helpful comments on a draft manuscript. 


\section{REFERENCES}

Bannigan A, Lizotte-Waniewski M, Riley M, Baskin TI. 2008. Emerging molecular mechanisms that power and regulate the anastral mitotic spindle of flowering plants. Cell Motil Cytoskel 65: 1-11.

Cameron LA, Yang G, Cimini D, Canman J, Kisurina-Evgenieva O, Khodjakov A, Danuser G, Salmon ED. 2006. Kinesin 5-independent poleward flux of kinetochore microtubules in PtK1 cells. J. Cell Biol. 173: 173-179

Civelekoglu-Scholey G, Sharp D J, Mogilner A, Scholey JM. 2006. Model of chromosome motility in Drosophila embryos: adaptation of a general mechanism for rapid mitosis. Biophys J 90: 3966-3982

Cramer LP. 1999. Role of actin-filament disassembly in lamellipodium protrusion in motile cells revealed using the drug jasplakinolide. Current Biology 9: 1095-1105.

Czaban BB, Forer A. 1994. Rhodamine-phalloidin and anti-tubulin antibody staining of spindle fibres that were irradiated with an ultraviolet microbeam. Protoplasma 178: 18-27.

Fabian L, Forer A. 2005. Redundant mechanisms for anaphase chromosome movements: cranefly spermatocyte spindles normally use actin filaments but also can function without them. Protoplasma 225: 169-184. 
Fabian L, Forer A. 2007. Possible roles of actin and myosin during anaphase chromosome movements in locust spermatocytes. Protoplasma 231: 201-213

Fabian L, Troscianczuk, J, Forer A. 2007a. Calyculin A, an enhancer of myosin, speeds up anaphase chromosome movement. BMC Cell and Chromosome 6:1 (17 pages).

Fabian L, Xia X, Venkitaramani DV, Johansen KM, Johansen J, Andrew DJ, Forer A. 2007b. Titin in insect spermatocyte spindle fibers associates with microtubules, actin, myosin and the matrix proteins skeletor, megator and chromator. J Cell Sci 120: 2190-2204.

Forer A. 1966. Characterization of the mitotic traction system, and evidence that birefringent spindle fibers neither produce nor transmit force for chromosome movement. Chromosoma 19: 44-98.

Forer A, Fabian L. 2005. Does 2,3-butanedione monoxime inhibit nonmuscle myosin? Protoplasma 225: 1-4.

Forer A, Pickett-Heaps JD. 1998. Cytochalasin D and latrunculin affect chromosome behaviour during meiosis in crane-fly spermatocytes. Chromosome Res 6: 533-549. 
Forer A, Pickett-Heaps JD. 2005. Fibrin clots keep non-adhering living cells in place on glass for perfusion or fixation. Cell Biol Int 29:721-730.

Forer A, Spurck T, Pickett-Heaps JD, Wilson PJ. 2003. Structure of kinetochore fibres in crane-fly spermatocytes after irradiation with an ultraviolet microbeam: neither microtubules nor actin filaments remain in the irradiated region. Cell Motil Cytoskeleton 56: 173-192.

Forer A, Spurck T, Pickett-Heaps JD. 2007. Actin and myosin inhibitors block elongation of kinetochore fibre stubs in metaphase crane-fly spermatocytes. Protoplasma 232: 79-85.

Forer A, Spurck T, Pickett-Heaps JD. 2008. What causes flux of tubulin in kinetochore microtubules? Protoplasma in press.

Gadde S, Heald R. 2004. Mechanisms and molecules of the mitotic spindle. Current Biology 14: R797-R805.

Holzinger A. 2001. Jasplakinolide. An actin-specific reagent that promotes actin polymerization. Methods Molec Biol 161: 109-120.

Holzinger A, Meindl U. 1997. Jasplakinolide, a novel targeting peptide, inhibits cell growth and induces actin filament polymerization in the green alga Micrasterias. Cell Motil Cytoskel 38: 365-372. 
Ilagan AB, Forer A. 1997a. The effects of UV-microbeam irradiation of kinetochores in cranefly spermatocytes. Cell Motil Cytoskel 36: 266-275.

Ilagan AB, Forer A. 1997b. Backwards chromosome movement in anaphase, after irradiations of kinetochores or kinetochore fibres. Protoplasma 198: 20-26.

Johansen KM, Johansen J. 2007. Cell and molecular biology of the spindle matrix. Intern Rev Cytol 263: 155-206.

Khodjakov A, Copenagle L, Gordon MB, Compton DA, Kapoor TM. 2003. Minus-end capture of preformed kinetochore fibers contributes to spindle morphogenesis. J Cell Biol 160: 671683.

LaFountain Jr JR, Janicke MA, Balczon R, Rickards GK. 1992. Cytochalasin induces abnormal anaphase in crane-fly spermatocytes and causes altered distribution of actin and centromeric antigens. Chromosoma 101:425-441.

LaFountain JR Jr, Oldenbourg R, Cole RW, Rieder CL. 2001. Microtubule flux mediates poleward motion of acentric chromosome fragments during meiosis in insect spermatocytes. Mol Biol Cell 12: 4054-4065. 
LaFountain JR, Cole RW, Rieder CL. 2002. Partner telomeres during anaphase in crane-fly spermatocytes are connected by an elastic tether that exerts a backward force and resists poleward movement. J Cell Sci 115: 1541-1549.

Lénárt P, Bacher CP, Daigle N, Hand AR, Eils R, Terasaki M, Ellenberg J. 2005. A contractile nuclear actin network drives chromosome congression in oocytes. Nature 436: 812-818.

Maiato H, Rieder CL, Khodjakov A. 2004. Kinetochore-driven formation of kinetochore fibers contributes to spindle assembly during animal mitosis. J Cell Biol 167: 831-840.

McNally K, Audhya A, Oegama K, McNally FJ. 2006. Katanin control mitotic and meiotic spindle length. J Cell Biol 175: 881-891.

Mitchison TJ, Maddox P, Gaetz J, Groen A, Shirasu M, Desai A, Salmon ED, Kapoor TM. 2005. Roles of polymerisation dynamics, opposed motors, and a tensile element in governing the length of Xenopus extract meiotic spindles. Molec Biol Cell 16: 3064-3076

Miyamoto, DT, Perlman ZE, Burbank, KS, Groen AC, Mitchison TJ. 2004. The kinesin Eg5 drives poleward microtubule flux in Xenopus laevis egg extract spindles J Cell Biol 167: 813818

O’Connell CB, Khodjakov AL. 2007. Cooperative mechanisms of mitotic spindle formation. J Cell Sci 120: 1717-1722. 
Osborn M, Weber K. 1982. Immunofluorescence and immunocytochemical procedures with affinity purified antibodies: tubulin containing structures. Methods Cell Biol 24: 97-132.

Qi H, Ding Y, Zhu L, Rath U, Johansen KM, Johansen J. 2007. Mutational analysis of the role of spindle matrix proteins in tubulin spindle assemble and function., American Society for Cell Biology Meeting, December 2007, Abstract 1481.

Robinson RW, Snyder J A. 2005. Localization of myosin II to chromosome arms and spindle fibers in PtK1 cells: a possible role for an actomyosin system in mitosis. Protoplasma 225: 113122.

Rogers GC, Rogers SL, Schwimmer TA, Ems-McClung SC, Walczak CE, Vale RD, Scholey JM, Sharp DJ. 2004. Two mitotic kinesins cooperate to drive sister chromatid separation during anaphase. Nature 427: 364-370.

Schaap CJ, Forer A. 1979. Temperature effects on anaphase chromosome movement in the spermatocytes of two species of crane flies (Nephrotoma suturalis Loew and Nephrotoma ferruginea Fabricius). J Cell Sci 39: 29-52.

Scholey JM, Rogers GC, Sharp DJ. 2001. Mitosis, microtubules, and the matrix. J Cell Biol 154: $261-266$ 
Silverman-Gavrila RV, Forer A. 2000. Evidence that actin and myosin are involved in the poleward flux of tubulin in metaphase kinetochore microtubules of crane-fly spermatocytes. J Cell Sci 113: 597-609.

Silverman-Gavrila R, Forer A. 2001. Effects of anti-myosin drugs on anaphase chromosome movement and cytokinesis in crane-fly primary spermatocytes. Cell Motil Cytoskel 50: 180197.

Silverman-Gavrila RV, Forer A.2003. Myosin localization during meiosis I of crane-fly spermatocytes gives indications about its role in division. Cell Motil Cytoskel 55: 97-113.

Spurck TP, Stonington OG, Snyder JA, Pickett-Heaps JD, Bajer A, Mole-Bajer J. 1990. UV microbeam irradiations of the mitotic spindle. II Spindle fiber dynamics and force production. $\mathrm{J}$ Cell Biol 111: 1505-1518.

Spurck T, Forer A, Pickett-Heaps J. 1997. Ultraviolet microbeam irradiations of epithelial cell and spermatocyte spindles suggest that forces act on the kinetochore fibre and are not generated by its disassembly. Cell Motil Cytoskel 36: 136-148.

Terada Y, Simerly C, Schatten G. 2000. Microfilament stabilization by jasplakinolide arrests oocyte maturation, cortical granule exocytosis, sperm incorporation, cone resorption, and cellcycle progression, but not DNA replication, during fertilization in mice. Molec Reproduct Devt 56: 89-98. 
Wadsworth P, Khodjakov A. 2004. E pluribus unum: towards a universal mechanism for spindle assembly. Trends Cell Biol 14: 413-419.

Wong R, Forer A. 2003. 'Signalling' between chromosomes in living crane-fly spermatocytes studied using ultrviolet microbeam irradiation. Chrom Res 11: 771-786.

Wong R, Forer A. 2004. Backward chromosome movement in crane-fly spermatocytes after UV microbeam irradiation of the interzone and a kinetochore. Cell Biol Intern 28: 293-298.

Yang G, Houghtaling BR, Gaetz J, Liu JZ, Danauser G, Kapoor TM. 2007. Architectural dynamics of the meiotic spindle revealed by single fluorophore imaging. Nature Cell Biol 9: 1233-1242.

Yasuda H, Kanda K, Koiwa H, Suenaga K, Kidou S, Ejiri S. 2005. Localization of actin filaments on mitotic apparatus in tobacco BY-2 cells Planta 222: 118-129.

Yin B, Forer A. 1996. Coordinated movements between autosomal half-bivalents in crane-fly spermatocytes: evidence that 'stop signals’ are sent between partner half-bivalents. J Cell Sci 109:155-163. 
TABLE I: Jasplakinolide added during anaphase.

Chromosome

movement

Stopped

Slowed

Accelerated

No effect
Number of

separating pairs Percentage

16

30

25

47

1

2

11

21 
TABLE II: Chromosome movements while kept in jasplakinolide

\begin{tabular}{lll}
$\begin{array}{l}\text { Initial effect of } \\
\text { jasplakinolide }\end{array}$ & Numbers of & Numbers of pairs \\
on chromosome & pairs & for which chromosome \\
movement & & movements recovered \\
\hline
\end{tabular}

Slowed

Stopped
9

9
0

$8^{*}$

*: 1 pair was not followed long enough to be certain whether it did or did not recover 
TABLE III: Chromosome movements after washing out the jasplakinolide

\section{Effect of \\ jasplakinolide}

on chromosome

movements

\author{
Number of \\ chromosome pairs \\ Movement after washout
}

Slower Same Faster
Slowed

Stopped
16

7
3

11

2

0

0

$5^{*}$

*: 2 pairs resumed motion before washout 
TABLE IV: Jasplakinolide applied before anaphase versus during anaphase

\author{
Effect on anaphase \\ movements of pairs \\ of half-bivalents
}

Jasplakinolide added

Before anaphase

onset (percentage

out of 21 pairs)

\begin{abstract}
After anaphase
onset (percentage

out of 53 pairs)
\end{abstract}

No effect

$67 \%$

$21 \%$

Slow, or stop

(or accelerate)

$33 \%$

$79 \%$ 


\section{FIGURE LEGENDS}

Figure 1 illustrates a control cell from metaphase through autosomal telophase. The times at the top of each frame are in hours:minutes:seconds. Two bivalents are illustrated (12:54:40). Anaphase started shortly before 12:56:00. The two univalent sex chromosomes remain at the equator as the half-bivalents move poleward; they start to move poleward (white arrows) after the autosomes near the poles. Bar: $10 \mu \mathrm{m}$.

Figure 2 is a graph of distance between kinetochores (ordinate) versus time (abscissa) for the control cell illustrated in Figure 1. One bivalent (and half-bivalent pair) is indicated by + and the other by $\mathbf{O}$. The dashed line is the computer-generated line-of-best-fit for the indicated circles. The slope of the line, the velocity by which the half-bivalents separate, is $2.95 \mu \mathrm{m} / \mathrm{min}$.

Figure 3 illustrates a cell treated with jasplakinolide in early anaphase; both pairs of separating half-bivalents slowed when jasplakinolide was added (as seen in the graph of this cell in Figure 4A). The times at the top of each frame are in hours:minutes:seconds. Anaphase started shortly before 12:55:28. Jasplakinolide was added at 12:57:17 and was washed out at 13:06:30. Illustrations are labelled JASP when the cells were immersed in jasplakinolide; notlabelled cells were in Ringers solution. The white arrows indicate the two sex chromosomal univalents that started to segregate. The bar indicates $10 \mu \mathrm{m}$.

Figure 4 illustrates graphs of distance between kinetochores of separating halfbivalents (ordinate) versus time (abscissa) for different cells treated with jasplakinolide. In the cell shown in Figure 4A (the same cell illustrated in Figure 3), jasplakinolide was added as indicated by the leftmost vertical dashed line, and was washed out as indicated by the rightmost 
vertical dashed line. The solid line is the line of best fit for the points in anaphase prior to addition of jasplakinolide, for the pair indicated by + , and the dashed lines are the lines of best fit for both sets of points after addition of jasplakinolide. Both pairs of chromosomes slowed after japlakinolide was added and did not change speed when the jsaplakinolide was washed out. In the cell shown in Figure 4B, jasplakinolide was added as indicated by the vertical dashed line, and the cell remained in jasplakinolide after that. The dashed line is the line of best fit for the points (in anaphase) prior to addition of jasplakinolide. The pair of chromosomes stopped separating after jasplakinolide was added, but resumed movement again after about 9 minutes. In the cell shown in Figure 4C, jasplakinolide was added as indicated by the leftmost vertical dashed line, and was washed out as indicated by the rightmost vertical dashed line. The solid line is the line of best fit for the points prior to adding jasplakinolide, and the dashed line is the line of best fit for the points after the jasplakinolide was washed out. The chromosome pair stopped separating when jasplakinolide was added and resumed movement after jasplakinolide was washed out.

Figure 5 illustrates the extent of slowing (as a percentage of initial velocity) for those chromosome pairs that slowed down after addition of jasplakinolide.

Figure 6 illustrates the extent of slowing (as a percentage of initial velocity), ordinate, as a function of the initial velocity of separation (abscissa) for those chromosome pairs that slowed down after addition of jasplakinolide. The dashed line is the computer-generated lineof-best-fit through the points.

Figure 7 includes all chromosome pairs that stopped moving when jasplakinolide was added. It illustrates the resumed velocity of separation as percent of initial velocity (ordinate) as a function of initial separation velocity (abscissa). 
Figure 8 illustrates effects on anaphase movements when jasplakinolide was added prior to anaphase. The graph indicates the effects on anaphase movements (abscissa) as a function of the time before anaphase when jasplakinolide was added (ordinate). For each cell in which the bivalent pairs were affected the same way, the two chromosome pairs measured are indicated by $\mathbf{O}$ and +, in the same colour; for clarity, the two points from each cell are slightly displaced from each other along the Y-axis (ordinate). When the bivalent pairs in the same cell responded differently, each pair was indicated by a solid circle in the same colour.

Figure 9 illustrates control cells stained for actin and tubulin; each non-merged image consists of the superposed images from three individual Z-series images. Phalloidin (the actin stain) stains the cell cortex, the spindle pole and the chromosomes prominently and the chromosome spindle fibres less prominently. In the merged image, actin is red, tubulin green, and co-staining yellow. The top cell (A-C) is in metaphase, the bottom cell (D-F) is in anaphase. The inset in E is the cell as seen in the confocal microscope using DIC microscopy. Each bar represents $10 \mu \mathrm{m}$.

Figure 10 illustrates an anaphase cell treated with jasplakinolide for 5 minutes and then lysed and stained for actin and tubulin. Each non-merged image consists of the superposed images from two individual Z-series images. In the merged image, actin is red, tubulin green, and co-staining yellow. Actin stains prominently the chromosomal spindle fibres, the chromosomes, and the pole. The bar represents $10 \mu \mathrm{m}$. 


\section{FIGURE 1}
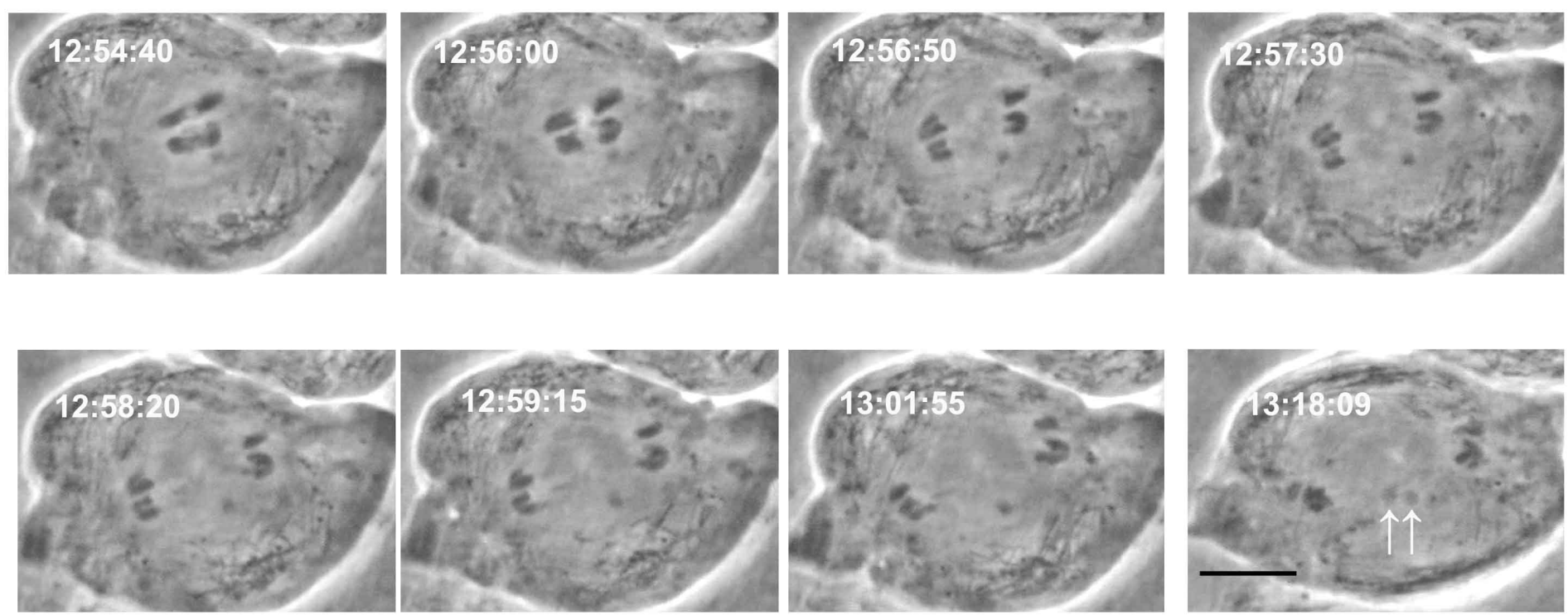
Figure 2

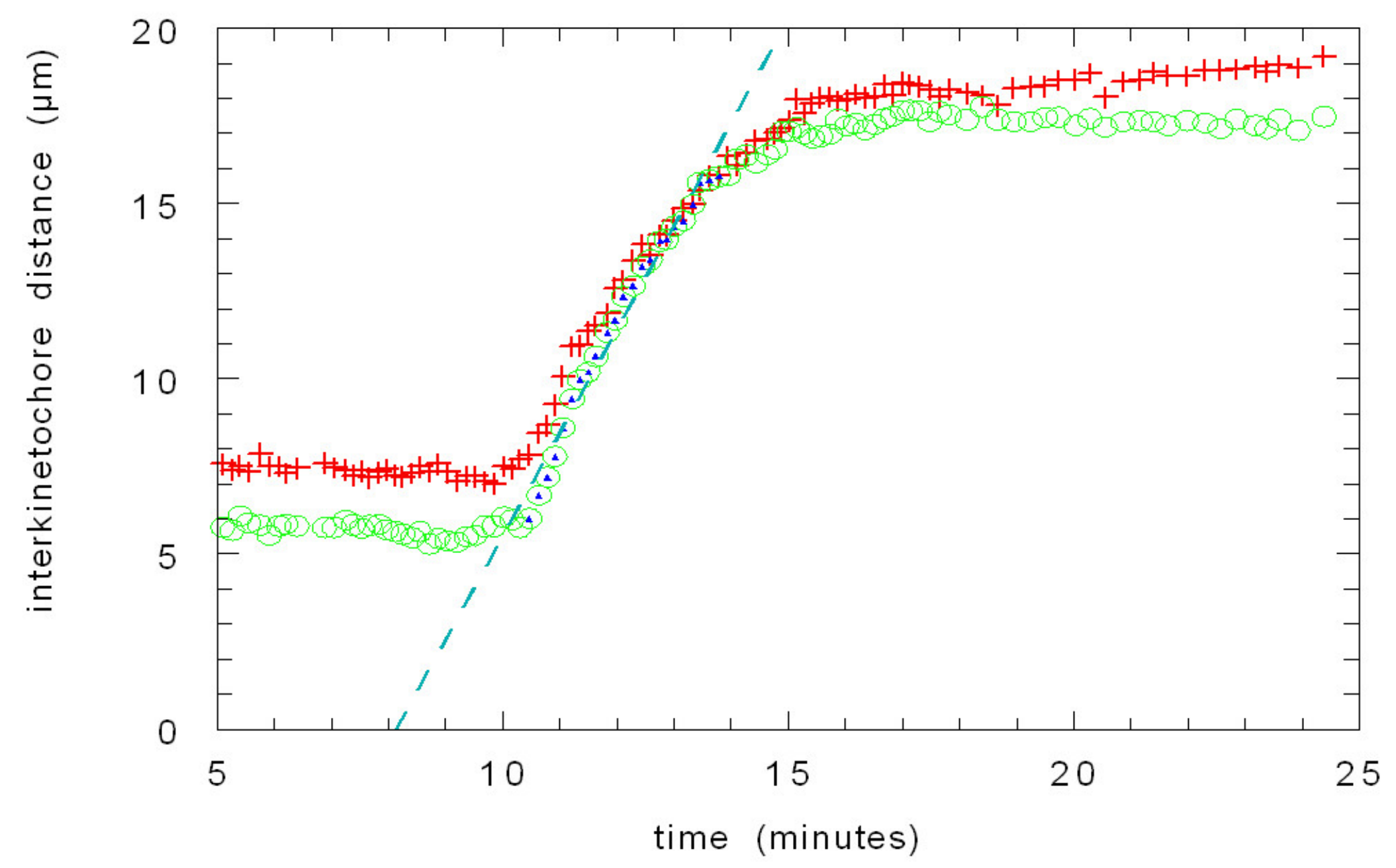


FIGURE 3

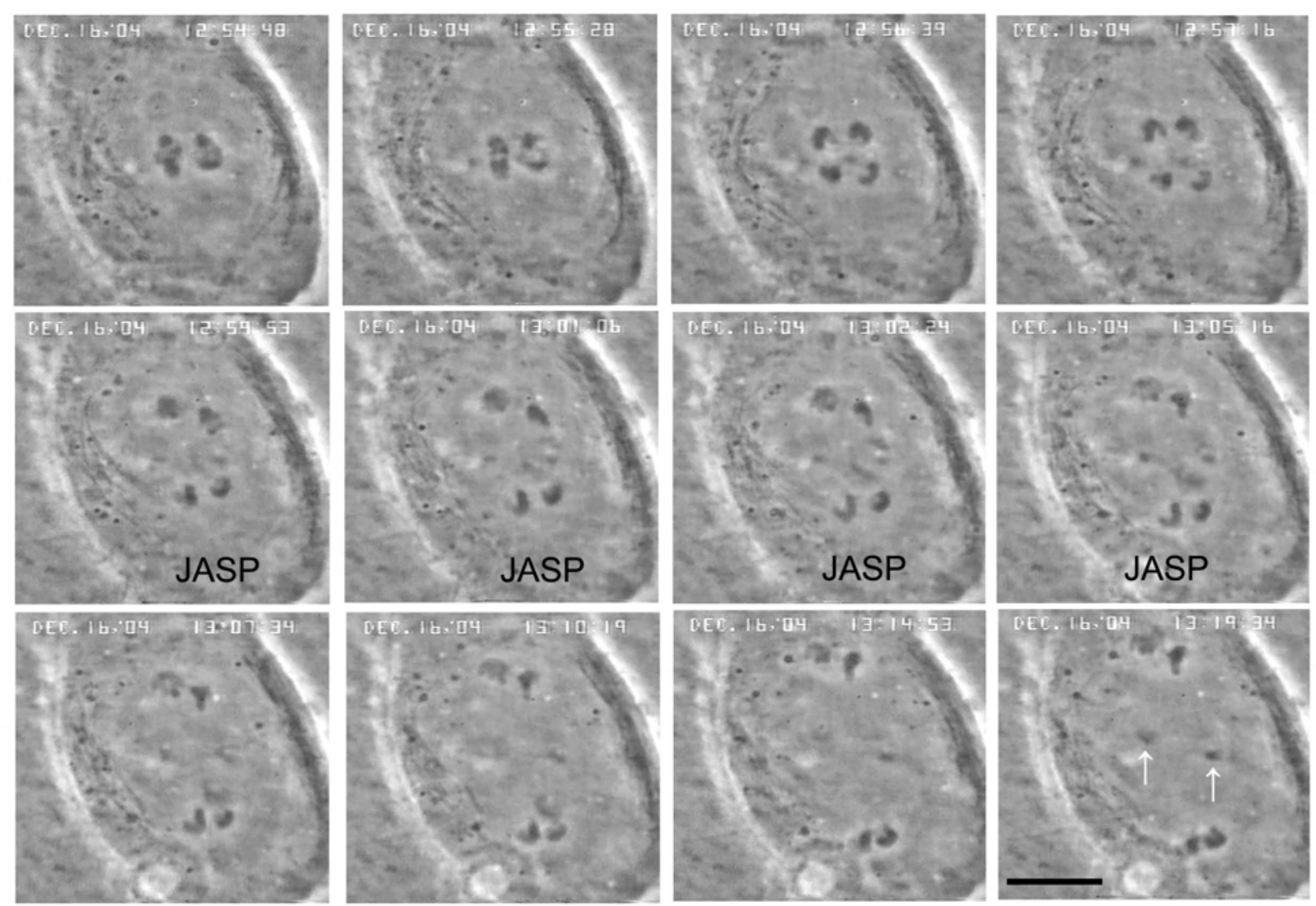


Figure $4 \mathrm{~A}$

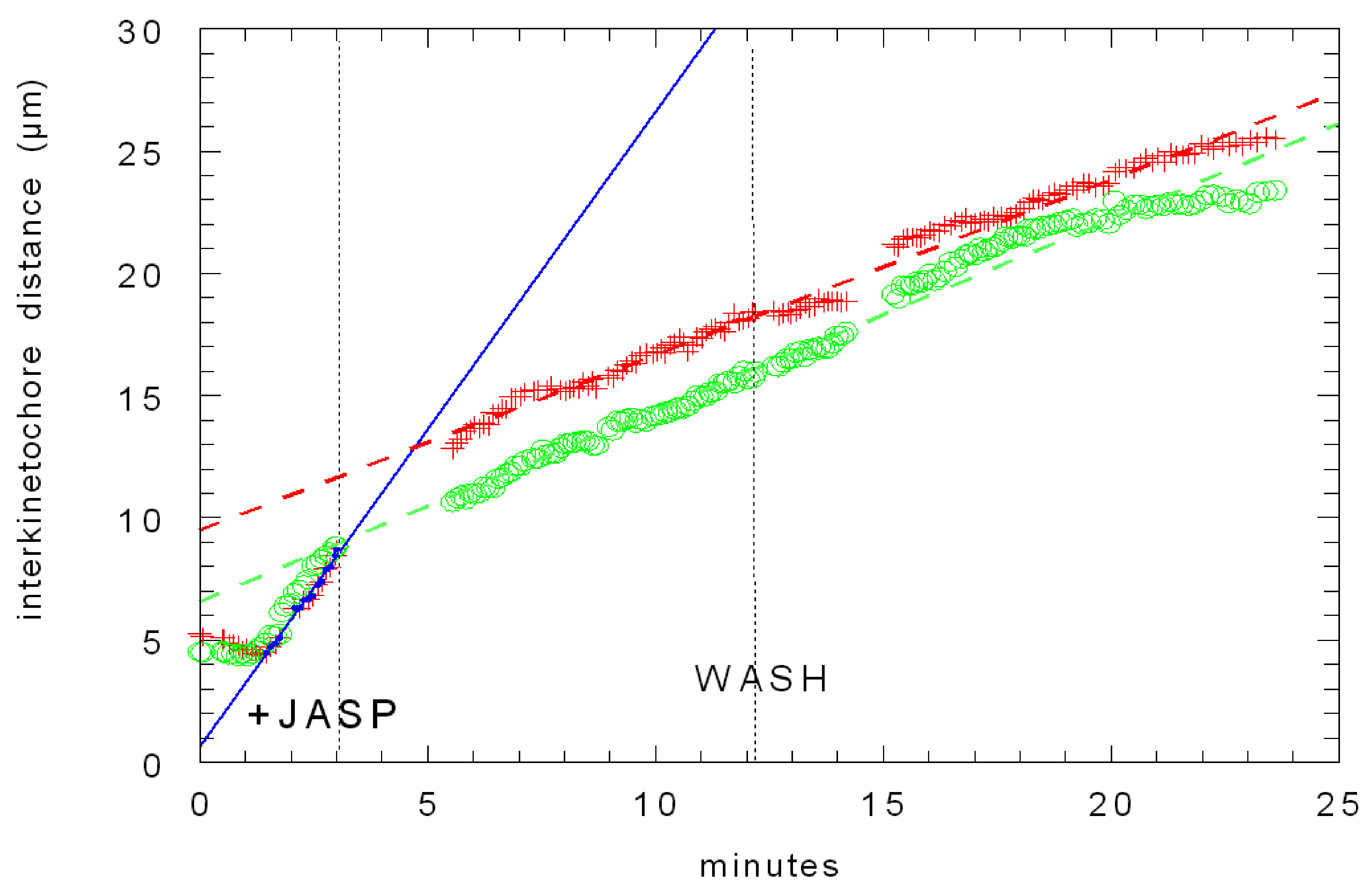


Figure 4B

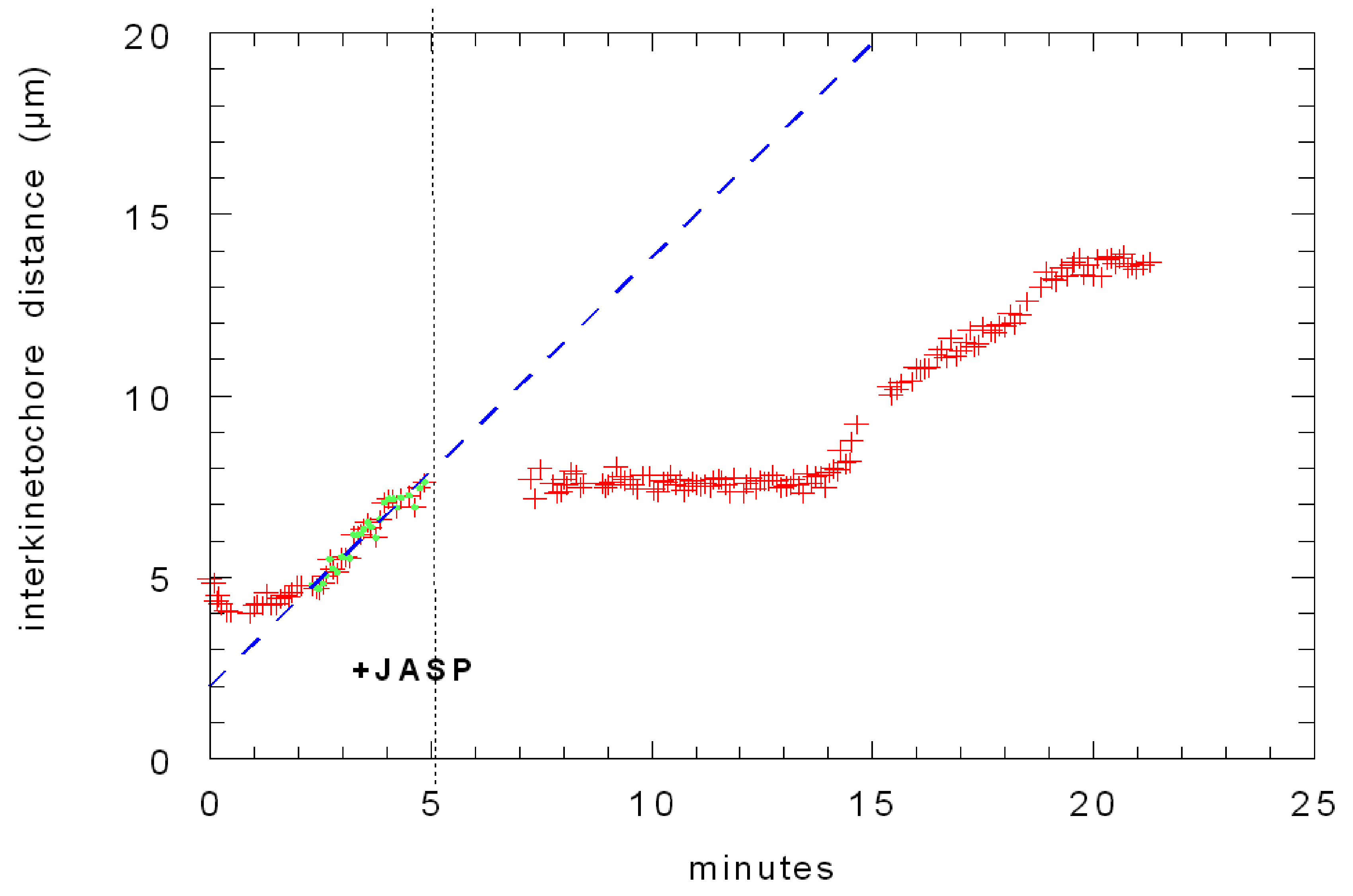


Figure $4 \mathrm{C}$

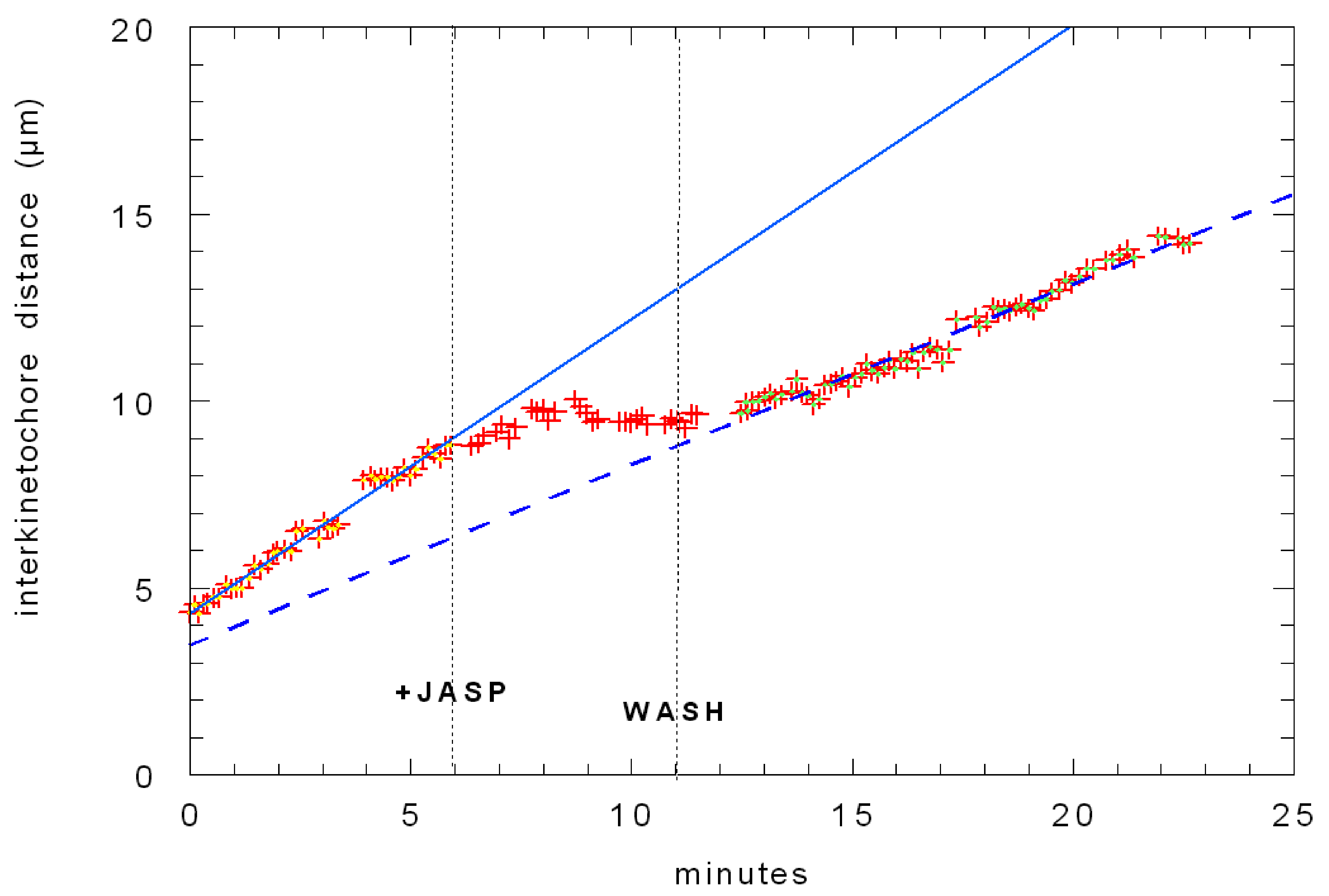




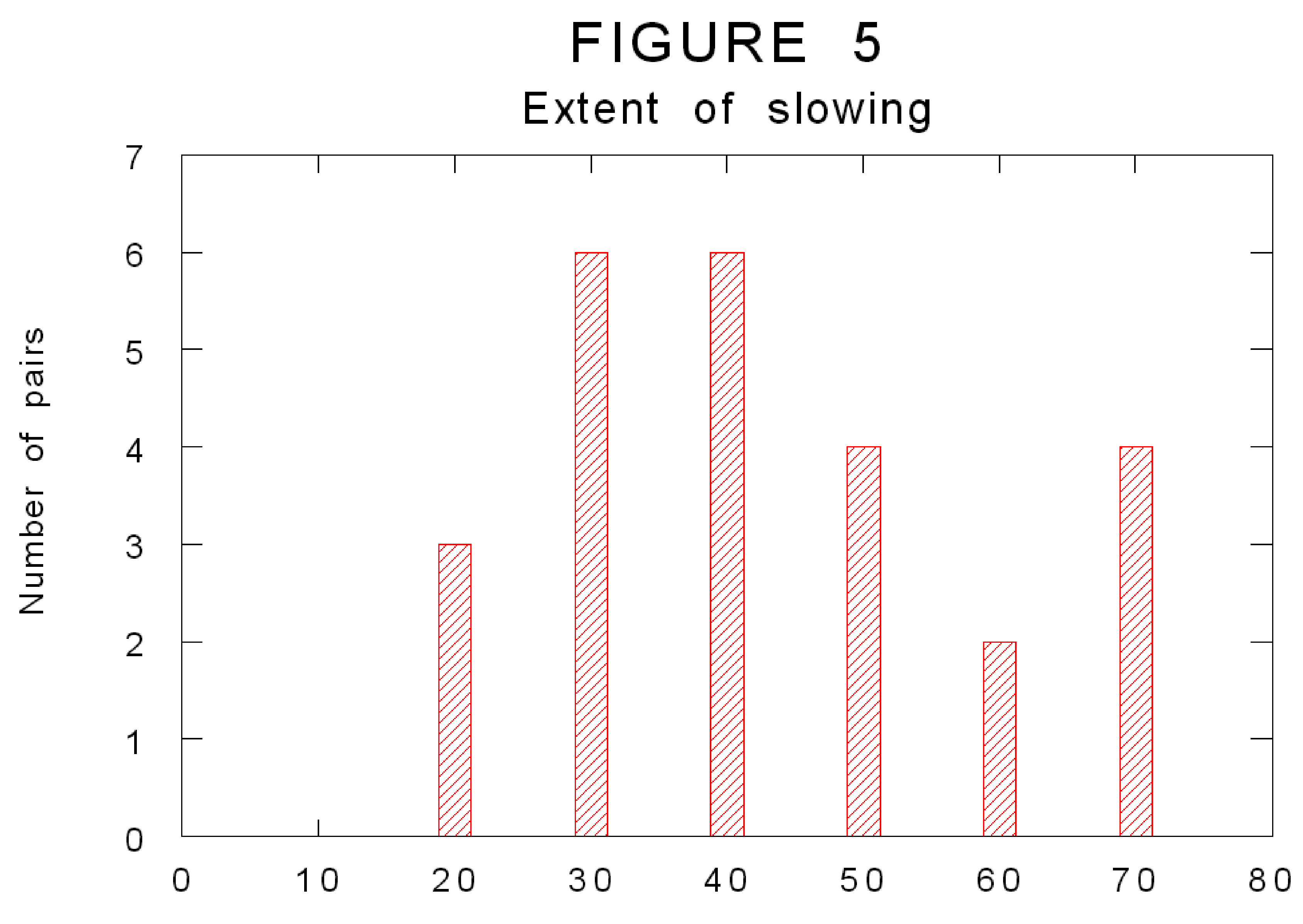

Final velocity as percent of initial velocity 
FIGURE 6

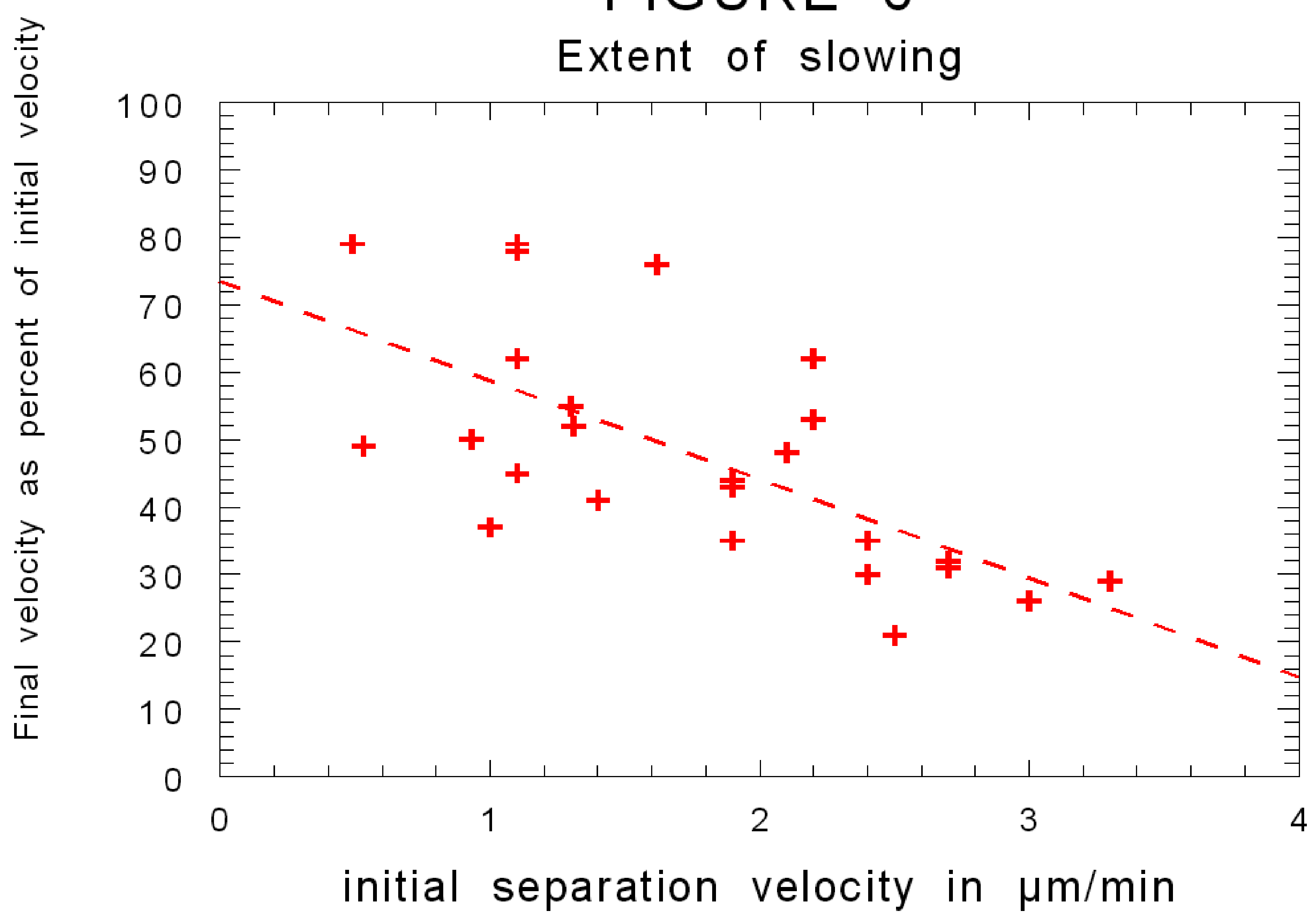




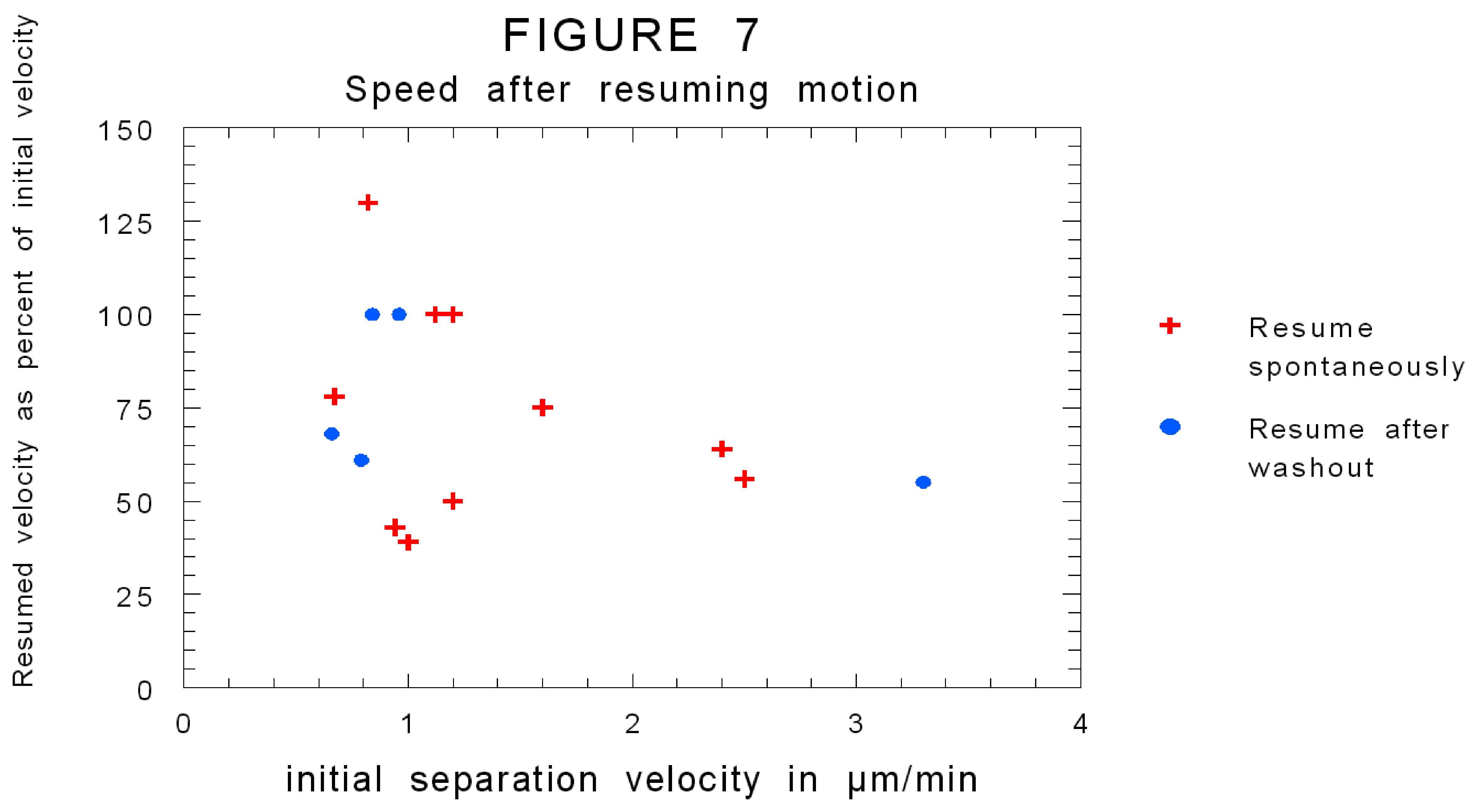




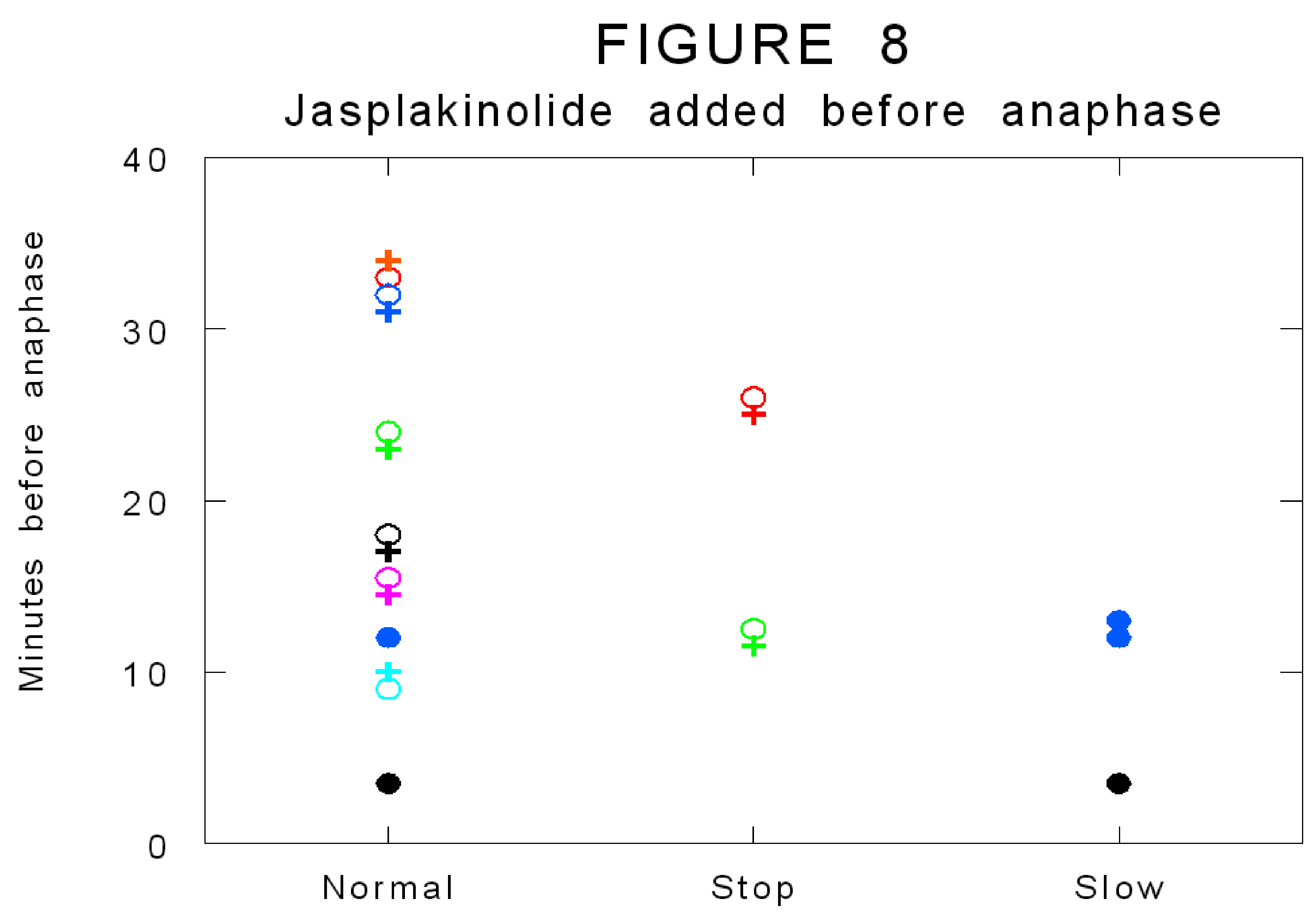

Effect on anaphase movements of half-bivalent pairs 


\section{FIGURE 9}

Actin
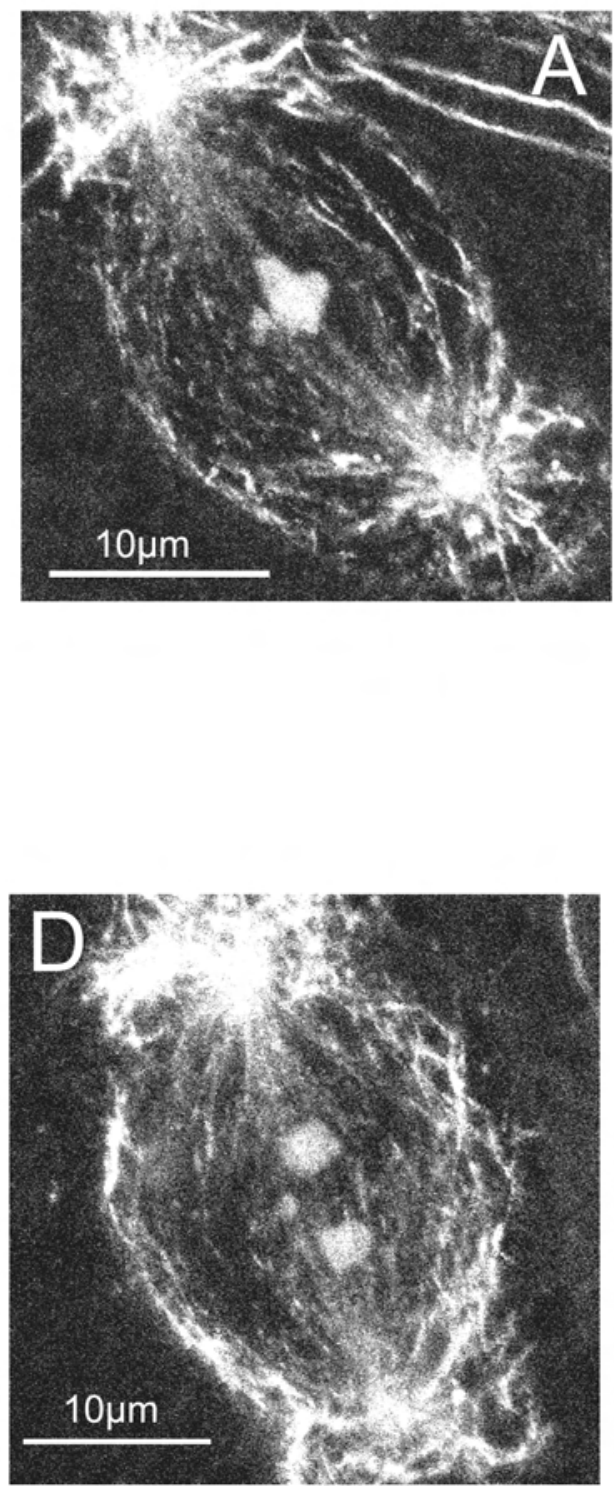

Tubulin

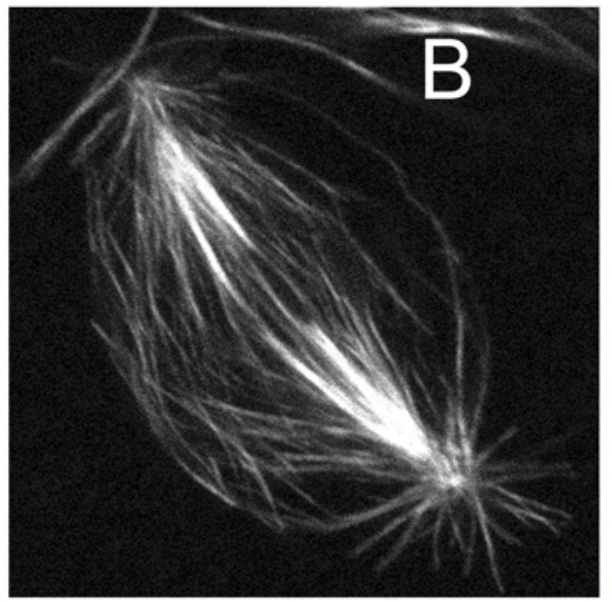

Merged

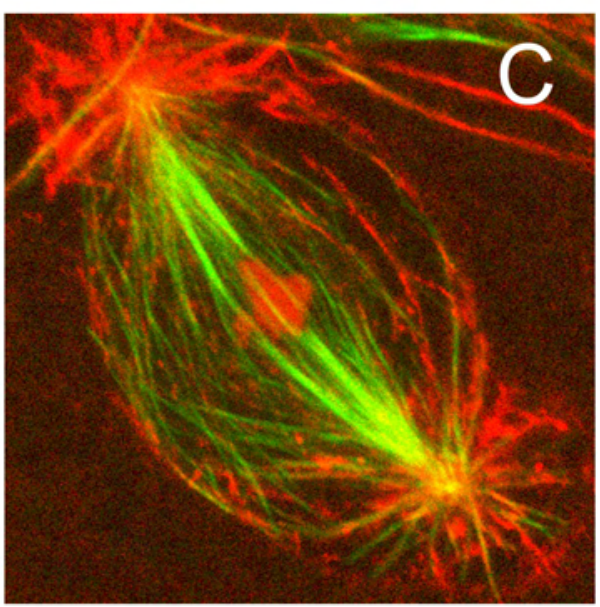

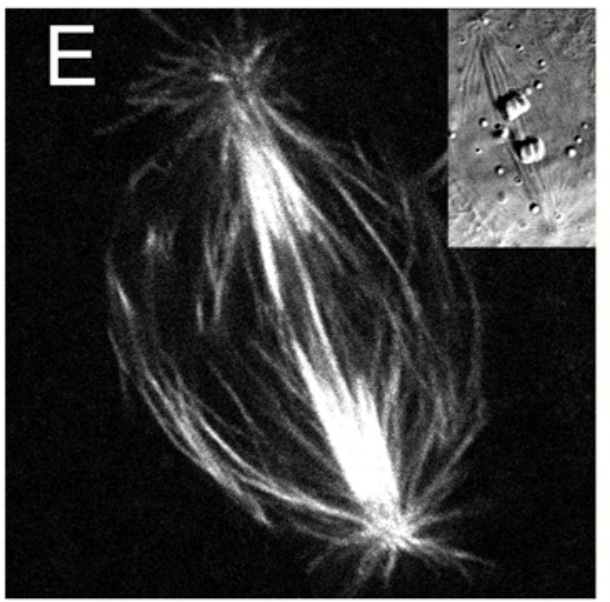

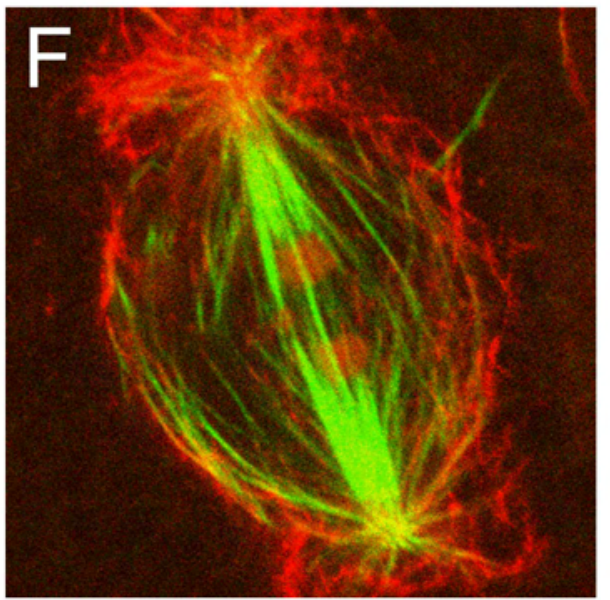




\section{FIGURE 10}

Actin

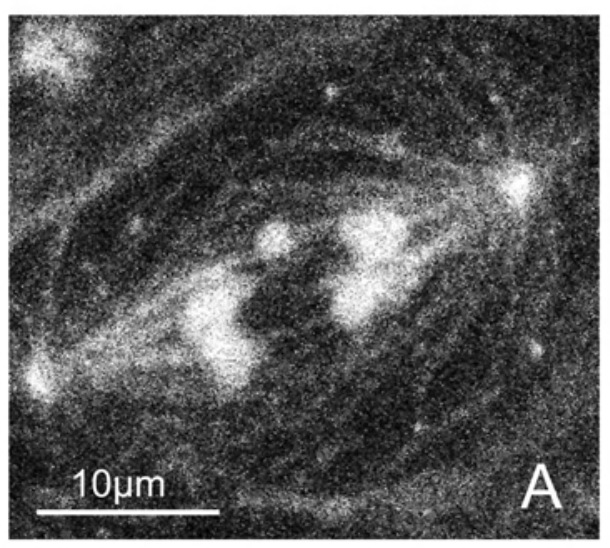

Tubulin

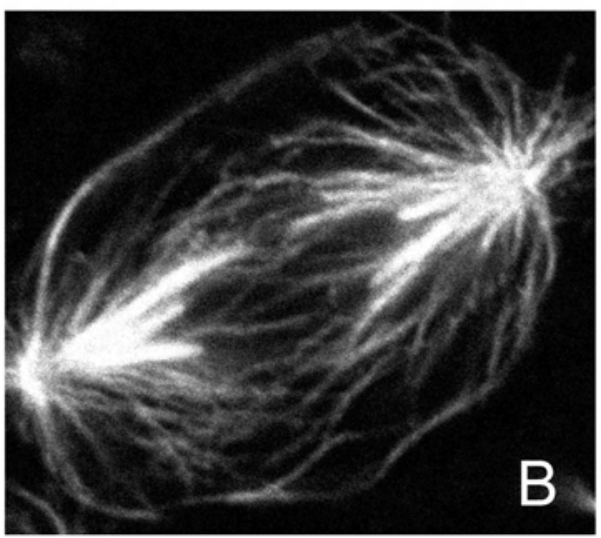

Merged

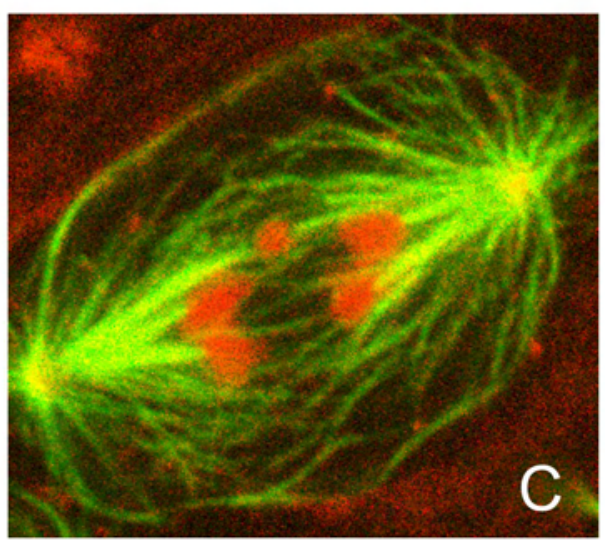

